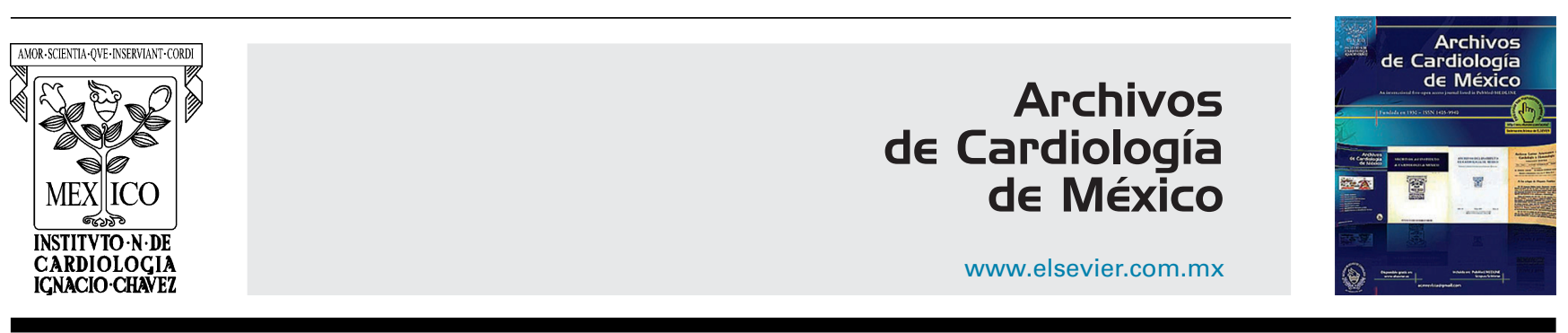

ARTÍCULO ESPECIAL

\title{
Hipertensión arterial pulmonar asociada al virus de inmunodeficiencia humana. A 3 décadas de su descripción
}

\section{José Luis Sandoval Gutiérrez*}

Jefatura de Áreas Críticas, Instituto Nacional de Enfermedades Respiratorias, Ciudad de México, México

Recibido el 19 de julio de 2017; aceptado el 8 de marzo de 2018

\section{PALABRAS CLAVE \\ Hipertensión arterial pulmonar; \\ $\mathrm{VIH}$; \\ Circulación \\ pulmonar; \\ México}

\section{KEYWORDS}

Pulmonary arterial

hypertension;

HIV;

Pulmonary

circulation;

Mexico

\begin{abstract}
Resumen El desarrollo de hipertensión arterial pulmonar asociada al virus de inmunodeficiencia humana reduce la probabilidad de sobrevivencia en el paciente afectado en comparación con el que no presenta esta alteración cardiopulmonar. La fisiopatogenia aún es incierta. Existen varias líneas de investigación para asociar las diferentes proteínas del virus en la lesión endotelial. Desde el punto de vista terapéutico, existen modalidades de tratamiento que permiten una expectativa de vida aceptable.

(c) 2018 Instituto Nacional de Cardiología Ignacio Chávez. Publicado por Masson Doyma México S.A. Este es un artículo Open Access bajo la licencia CC BY-NC-ND (http://creativecommons. org/licenses/by-nc-nd/4.0/).
\end{abstract}

Pulmonary arterial hypertension associated with human immunodeficiency virus: thirty years after its description

\begin{abstract}
The development of pulmonary arterial hypertension associated with human immunodeficiency virus reduces the probability of survival in the patient affected compared to those without cardiopulmonary disease. The pathophysiology is uncertain. There are several lines of research to associate the different proteins of the virus in the endothelial lesion. From a therapeutic point of view there are treatment modalities that allow an acceptable life expectancy.

(c) 2018 Instituto Nacional de Cardiología Ignacio Chávez. Published by Masson Doyma México S.A. This is an open access article under the CC BY-NC-ND license (http://creativecommons. org/licenses/by-nc-nd/4.0/).
\end{abstract} * Autor para correspondencia. Ismael Cosío Villegas, Tlalpan 4502 Col Sec XVI, CP 14080, CDMX. Teléfono: +54871700 ext 5226.
Correo electrónico: sandovalgutierrez@gmail.com 


\section{Introducción}

En 1987, Kim y Factor $^{1}$, a través del estudio de necropsia, describieron el primer caso histopatológico con datos compatibles de hipertensión arterial pulmonar (HAP) en un paciente fallecido por complicaciones del virus de inmunodeficiencia humana (VIH).

Este hallazgo en la circulación pulmonar, considerada la misma una enfermedad huérfana por su poca prevalencia en la población general, al presentarse en coexistencia al VIH motivó un campo amplio de investigación de esta dualidad.

La Organización Mundial de la Salud (OMS) clasifica a los pacientes con HAP en 5 grupos, siendo la HAP/VIH correspondiente al grupo $1^{2}$ (tabla 1$)$.

La HAP se define como una presión arterial media de la pulmonar $\geq 25 \mathrm{mmHg}$ en reposo medida por cateterismo cardiaco derecho, con una presión capilar pulmonar (PCP) $\leq 15 \mathrm{mmHg}$ y resistencia pulmonar vascular $>3$ unidades Woods.

En la mayoría de los centros se confirma el diagnóstico por ecocardiografía (ECO) y posteriormente se programa para el cateterismo cardiaco derecho ${ }^{3}$.

La asociación HAP/VIH se considera una complicación pulmonar no infecciosa de los pacientes con este virus, el cual disminuye la calidad de vida y el pronóstico de sobrevida a pesar de lograr un control en la carga viral y en la cuenta de linfocitos tipo CD4.

\section{Epidemiología}

La población VIH tiene un riesgo aumentado de 2,500 veces de padecer HAP con respecto a la población general no $\mathrm{VIH}^{4}$.

La prevalencia en países industrializados es de alrededor del $0.5 \%$ de la población infectada ${ }^{5}$, pero en lugares como el continente africano se ha reportado una presentación del $8 \%^{6}$.

En México se está realizando el registro de HAP el cual demostrará la incidencia y prevalencia de esta patología en los diferentes grupos ${ }^{7}$.

Tabla 1 Clasificación de la OMS de HAP grupo 1

1.1 HAP idiopática
1.2 HAP hereditaria
1.2.1 BMPR2
1.2.2 ALK-1, ENG, SMAD9, CAv1, KCNK3
1.2.3 Desconocida
1.3 Inducida por drogas y toxinas
1.4 Asociada a:
1.4.1 Enfermedad del tejido conectivo
1.4.2 Infección por VIH
1.4.3 Hipertensión portal
1.4.4 Enfermedad cardiaca congénita
1.4.5 Esquistosomiasis

ALK-1: receptor activina quinasa tipo 1gene; BMPR2: receptor proteico morfogenético óseo tipo II; CAv1: caveolina-1; ENG: endoglina; HAP: hipertensión arterial pulmonar; KCNK3: subfamilia 3 canal de potasio; OMS: Organización Mundial de la salud; SMAD9: homólogo decapentaplégico tipo 9; VIH: virus de inmunodeficiencia humana.

\section{Diagnóstico clínico}

Las manifestaciones clínicas son similares a las otras formas de HAP, predominando la disnea de esfuerzo, la intolerancia al ejercicio y los datos de falla cardiaca derecha en fases avanzadas. Se observa disnea progresiva (85\%), edema periférico (30\%), tos no productiva (19\%), fatiga (13\%), presíncope y síncope (12\%) y dolor torácico $(7 \%)$.

En la mayoría de las ocasiones, a nivel global, se diagnostica la HAP/VIH en casos severos de la enfermedad, lo cual provoca que el manejo no sea exitoso, por lo que se enfatiza actualmente en considerar esta asociación en aquellos con disnea crónica y/o que no remiten mediante el manejo convencional.

\section{Diagnóstico de gabinete}

La ECO, la tomografía con angiografía pulmonar y la resonancia magnética han demostrado la misma utilidad que el resto de los grupos con HAP. Es imperante la realización del cateterismo cardiaco derecho para la medición de la hemodinámica correspondiente.

\section{Fisiopatogenia}

La patogénesis sigue siendo compleja. Esta se caracteriza por 3 procesos mayores: vasoconstricción, remodelamiento vascular y eventos microtrombóticos (tabla 2).

La infección por VIH induce secreción de algunas citoquinas por los monocitos, macrófagos y linfocitos (IL-1, IL-6, IL-8, IL-13,TNF), además del factor de crecimiento de plaquetas (PDGF); todo esto exacerba la inflamación del endotelio vascular, provocando en el paciente susceptible la aparición de HAP.

La IL-8 juega un papel importante en el desarrollo de HAP, especialmente en las fases tempranas del remodelamiento vascular, ya que tiene efecto proangiogénico y

Tabla 2 Fisiopatogenia de la HAP/VIH

Predisposición genética

HDL-DR6

HDA-DR5

Proteínas relacionadas con el VIH

Factor negativo

Proteínas de transcripción

Glicoproteína 120

Proteínas de inflamación relacionadas con el VIH

IL-1, IL-6, IL-8, IL-13, TNF-alfa, PDGF

Patogénesis

Eventos microtrombóticos

Vasoconstricción

Remodelamiento vascular

Histopatología

Arteriopatía pulmonar primaria

Enfermedad venooclusiva pulmonar

Hemangiomatosis capilar pulmonar

HAP: hipertensión arterial pulmonar; IL: interleucina; PDGF: factor de crecimiento derivado de plaquetas; VIH: virus de inmunodeficiencia humana. 
antiapoptótico. La IL-13 es un mediador importante en la proliferación celular y en el remodelamiento tisular pulmonar.

Las proteínas virales del VIH, como el factor negativo (Nef), de transcripción (Tat) y glicoproteína 120 (gp120), inducen estrés oxidativo vascular, proliferación del músculo liso y lesión endotelial. La proteína Tat estimula la actividad de las células endoteliales vía IL-6. La presencia de proteína Nef se asocia a lesiones plexiformes. En la vasculatura pulmonar esta proteína puede entrar en las células endoteliales vía receptor CXCR-4, provocando proliferación y apoptosis ${ }^{8}$.

La localización de esta proteína en los espacios lipídicos de la pared celular explica su participación y el tipo de lesión resultante.

El receptor proteico morfogenético óseo tipo II (BMPR2) participa en varios procesos: inhibición de la proliferación vascular del músculo liso, promoviendo la sobrevivencia de las células endoteliales, previniendo el daño de la respuesta inflamatoria por lo que la mutación de este receptor aumenta la sensibilidad a padecer HAP. La predisposición genética se ha relacionado también en presencia de HDL-DR6 y HLA-DR5.

Últimamente se ha descrito la participación de la endotelina en la fisiopatogenia de la HAP/VIH, lo cual abre un campo de investigación terapéutica con respecto a los agonistas de los receptores de esta proteína ${ }^{8,9}$.

\section{Histopatología}

Histológicamente existen las mismas manifestaciones que en el resto de las presentaciones de HAP. Predominan 3 presentaciones: arteriopatía pulmonar primaria (plexiforme, trombótica, hipertrofia de la media y fibrosis de la íntima), enfermedad pulmonar venooclusiva y hemangiomatosis capilar pulmonar ${ }^{10}$.

La forma más frecuente en la HAP/VIH es la arteriopatía plexogénica, seguido de arteriopatía pulmonar trombótica, hipertrofia de la media con fibrosis de la íntima y enfermedad venooclusiva ${ }^{11}$ (fig. 1).

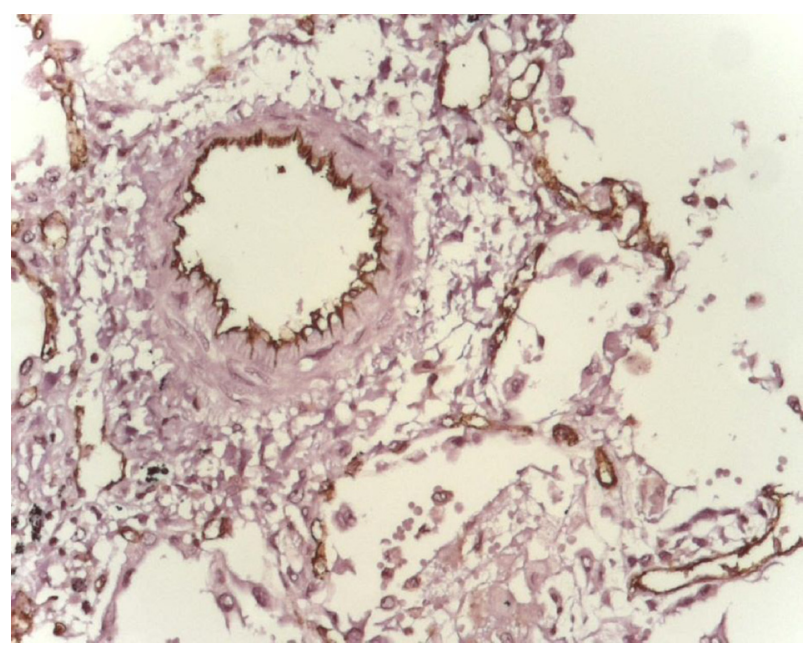

Figura 1 Expresión de endotelina-1 (color café) en paciente masculino en la $3 .^{a}$ década de la vida con $\mathrm{VIH}$, fallecido por complicaciones pulmonares.
Tabla 3 Tratamiento antirretroviral: grupos y medicamentos más característicos

Inhibidores de la transcriptasa reversa análogos de los nucleósidos

Abacavir, tenofovir, lamivudina, zidovudina

Inhibidores de la transcriptasa reversa análogos de los no

nucleósidos

Efavirenz

Inhibidores de proteasa

Atazanavir, indinavir, ritonavir, lopinavir, ritonavir

Inhibidores de fusión

Enfuvirtida

Inhibidores de integrasa

Raltegravir

Combinaciones de los diferentes grupos farmacológicos

\section{Tratamiento antirretroviral}

Hasta el momento no existe una cura para la HAP/VIH; es indispensable la utilización del tratamiento antirretroviral (ARV), ya que este tiene beneficios clínicos en el manejo de estos pacientes. Aunque se ha demostrado que el ARV en las fases iniciales de la HAP/VIH tiene efectos benéficos, faltan estudios prospectivos que demuestren el efecto del mismo en la hemodinámica de la circulación pulmonar ${ }^{12}$.

Actualmente existen más de 30 medicamentos de este tipo en el mercado farmacéutico (tabla 3 ).

Los pacientes que han recibido tenofovir han presentado menos prevalencia de $\mathrm{HAP} / \mathrm{VIH}$.

\section{Tratamiento para la hipertensión arterial pulmonar}

Actualmente se cuenta con herramienta terapéutica para manejar este binomio (tabla 4). Es de considerar que cuando se presenta falla cardiaca derecha, el tratamiento va orientado a lo que regularmente se maneja en esta situación.

Tabla 4 Tratamiento para HAP/VIH

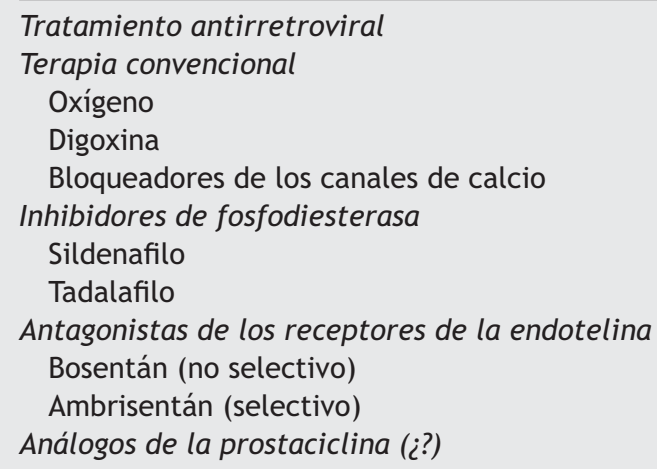

HAP: hipertensión arterial pulmonar; VIH: virus de inmunodeficiencia humana. 


\section{Conclusiones}

La prevalencia del VIH en la población general a nivel global se ha mantenido $(0.8 \% \text { de la población adulta })^{13}$, lo que obliga a buscar otras complicaciones no infecciosas, que pueden empeorar el pronóstico a pesar del control viral que se pueda tener. Las enfermedades cardiopulmonares tienen una presentación importante en este grupo de pacientes, a pesar de que el ARV permite una mayor sobrevida. La HAP/VIH hasta el momento ha pasado desapercibida por clínicos de primer contacto, internistas, infectólogos, neumólogos y cardiólogos ${ }^{14}$. Es necesaria la difusión de esta entidad para que el diagnóstico oportuno, el óptimo manejo y la mejoría de los pacientes se hagan una realidad.

\section{Financiación}

No se recibió patrocinio de ningún tipo para llevar a cabo este artículo.

\section{Conflicto de intereses}

El autor declara no tener ningún conflicto de intereses.

\section{Bibliografía}

1. Kim KK, Factor SM. Membranoproliferative glomerulonephritis and plexogenic pulmonary arteriopathy in a homosexual man with acquired immunodeficiency syndrome. Hum Pathol. 1987; 18:1293-6.

2. Simonneau G, Gatzoulis MA, Adatia I, et al. Updated clinical classification of pulmonary hypertension. J Am CollCardiol. 2013;62 25 Suppl.:D34-41.

3. Sandoval Gutiérrez JL, Vega RS, Murakami A, et al. Hipertensión arterial pulmonar asociada al virus de inmunodeficiencia humana. Una entidad emergente. Neumol Cir Torax. 2007;66:88-92.
4. Sandoval-Gutiérrez JL, Santos-Martínez LE, Rodríguez-Silverio $\mathrm{J}$, et al. Pulmonary arterial hypertension associated to human immunodeficiency virus. Arch Cardiol Mex. 2015;85:118-23.

5. leong $M$, Farber HW. Pulmonary arterial hypertension associated with human immunodeficiency virus. UpToDate [consultado 3 Ene 2018]. Disponible en: https://www.uptodate.com/ contents/pulmonary-arterial-hypertension-associated-withhuman-immunodeficiency-virus?source=search_result\&search= pulmonary\%20arterial\%20hypertension\%20and\%20hiv\&selected Title $=1 \sim 150$

6. Sliwa K, Carrington MJ, Becker A, et al. Contribution of the human immunodeficiency virus/acquired immunodeficiency syndrome epidemic to de novo presentations of heart disease in the Heart of Soweto Study cohort. Eur Heart J. 2012;33:866-74.

7. Sandoval Zarate J, Jerjes-Sanchez C, Ramirez-Rivera A, et al., REMEHIP Investigators. Mexican registry of pulmonary hypertension: REMEHIP. Arch Cardiol Mex. 2017;87:13-7.

8. Sandoval-Gutierrez JL, Rodriguez-Silverio J, Rivera-Rosales RM, et al. Overexpression of endothelin-1 leads to more severe pulmonary complex vascular lesions associated with the human immunodeficiency virus. Arch Med Res. 2015;46:228-32.

9. Parikh RV, Scherzer R, Nitta EM, et al. Increased levels of asymmetric dimethylarginine are associated with pulmonary arterial hypertension in HIV infection. AIDS. 2014;28:511-9.

10. Mehta NJ, Khan IA, Mehta RN, et al. HIV-related pulmonary hypertension: analytic review of 131 cases. Chest. 2000;118:1133-4.

11. Pietra GG, Edwards WD, Kay JM, et al. Histopathology of primary pulmonary hypertension. A qualitative and quantitative study of pulmonary blood vessels from 58 patients in the National Heart, Lung, and Blood Institute, Primary Pulmonary Hypertension Registry. Circulation. 1989;80:1198-206.

12. Cicalini S, Chinello P, Grilli E, et al. Treatment and outcome of pulmonary arterial hypertension in HIV-infected patients: a review of the literature. Curr HIV Res. 2009;7:589-96.

13. Global HIV and AIDS statistics [consultado 20 Ene 2018]. Disponible en: https://www.avert.org/global-hiv-and-aids-statistics

14. Sandoval-Gutiérrez JL. Pulmonary arterial hypertension associated with human immunodeficiency virus. The forgotten topic. Arch Cardiol Mex. 2014;84:54-5. 